\title{
Le Tramway de Claude Simon : une vanité postmoderne?
}

Le Tramway de Claude Simon, publié en 2001, quatre années avant le décès de son auteur à l'âge de quatre-vingt-deux ans, se voudrait être, non seulement comme le fait remarquer Mireille Calle-Gruber « un livre-testament » (Calle-Gruber, 2011, p. 426), mais il est plus que cela. Il est ce que nous proposons d'appeler une « autothanatographie $»^{1}$. Ce texte, qui reprend des faits advenus réellement à l'auteur, est, en quelque sorte, un dernier regard de l'écrivain sur sa vie et sur le monde.

À bien des égards, en effet, l'auteur, vieux et souffrant de plusieurs maux, notamment d'une insuffisance respiratoire (Calle-Gruber, 2011, p. 426), y exprime une profonde tristesse. Tous les thèmes, qui y sont développés, s'inscrivent dans le champ lexical de la mélancolie, en l'occurrence la maladie incurable de la mère, la mort de cette dernière suite à son cancer, la maladie de l'auteur et son hospitalisation en 1998, la guerre à laquelle il a pris part en tant que réserviste, la lente agonie de la nature, etc.

À cette mélancolie s'ajoute un "message » à forte coloration pessimiste, qui traverse le texte de bout en bout, mais en filigrane et que l'on pourrait interpréter comme le constat de l'auteur, à la fin de sa vie, de la vanité du monde que rien ne peut, désormais, sauver même pas la littérature.

Nous proposons dans le présent article d'analyser la nouvelle perception de la vanité du monde qui se dégage du Tramway de Claude Simon et les procédés usités dans ce sens pour l'instaurer. Notre travail s'articulera ipso facto autour de deux vo-

Mokhtar Belarbi - professeur de l'Enseignement Supérieur à La Faculté des Lettres et des Sciences Humaines de Meknès. Adresse pour correspondance : FLSH BP 11202 Zitoune- Meknès, CP. 50000. Maroc; e-mail : mokhtarbelarbi@yahoo.fr

1. Nous entendons par cette appellation, le récit autobiographique qui accorde une large part au thème de la mort. 
lets : dans le premier, que nous intitulons, memento mori, nous étudierons la portée de ce message "moralisateur" au sens postmoderne dans le texte de Claude Simon. Dans le second volet, que nous intitulons ars moriendi, nous analyserons une série d'images usitées par l'auteur pour évoquer la finitude tragique du monde.

\section{Memento mori}

Contrairement à ce que certains critiques ont avancé, Le Tramway de Claude Simon ne ressemble pas aux autres textes de ce dernier. Une certaine leçon "morale » est associée au thème de la mort qui y est fort dominant. L'auteur s'est toujours révolté contre l'idée que ses textes aient une quelconque visée didactique ou moralisatrice. «Absolument, affirme-t-il, aucune fonction ou vocation pédagogique dans mes livres. Aucune intention d'instituer ou d'insinuer un mode d'emploi » (Simon, 1998, p. 7). Avec Le Tramway, il semble que quelque chose s'est produite chez lui et qui l'a, de ce fait, poussé à changer d'avis à ce propos. En effet, il semble qu'il a fait sien cet adage des contemplateurs catholiques médiévaux et qu'ils consignaient sur leurs œuvres, à savoir memento mori. Soulignons que ces derniers entendaient par cette action rappeler aux êtres humains leur condition irrémédiable de mortels et qui, de ce fait, devaient s'abstenir de commettre des péchés. C'est pour cela que cette déclaration s'accompagne la plupart du temps de l'injonction biblique "In omnibus operibus tuis memorare novissima tua, et in aeternum non peccabis» (Siracide, 7: 36) ${ }^{2}$. Nous pensons que Claude Simon a fait pareil dans Le Tramway, à cette exception que ce rappel n'est sans doute pas destiné à son lecteur, mais il en serait plutôt luimême le destinataire et que le rapport entre la condition de mortel et le péché n'est pas mis en avant chez lui.

Ce qui nous permet de corroborer notre thèse est le nombre important d'éléments cités et qui sont d'une manière générale liés à la mort. Il ne s'agit guère de la conception gréco-romaine du memento mori associé au carpe diem, comme c'est le cas chez Horace. La conception simonienne est plutôt proche de la conception chrétienne sans l'épouser entièrement.

Dans Le Tramway, il n'y a, en effet, guère de références aux plaisirs de la vie. Même les plaisirs du corps, qui sont une constante dans l'œuvre de Claude Simon, ou les plaisirs de l'art, qui dans plusieurs textes de ce dernier transcendent les innombrables descriptions de plusieurs formes de douleur, sont inexistants. "J'ai été très souvent heureux (ce qui signifie que j'ai aussi été très malheureux, note-t-il, l'un ne va pas sans l'autre) dans ma vie, et de nombreuses façons, rapports affectifs et sexuels, lecture d'un bon livre (celle de Proust me plonge toujours dans un ravissement), contemplation de tableaux, d'architectures, écoute de musique, etc. » (cité

2. «Dans toutes tes actions souviens-toi de ta fin, et tu ne pécheras jamais ». 
par Calle-Gruber, 2011, p. 154). Or, il semble que ses plaisirs ne signifiaient plus rien à l'auteur, lorsqu'il s'est mis à écrire son dernier texte.

Dans Le Tramway, l'auteur, en effet, fait entrelacer plusieurs séquences, qui, dans l'ensemble, s'articulent autour de thèmes que l'on pourrait qualifier de noirs ou carrément lugubres. En d'autres termes, si une leçon de morale est à tirer de son texte, c'est que la mort est, telle l'épée de Damoclès, à l'affût. Elle est ce qui récupère tout. Elle est la dernière phase de la trajectoire que représente la Vie. L'omniprésence d'éléments, qui rappellent en permanence dans le texte l'imminence de son intervention, montre que l'auteur a tenu à la présenter comme une réalité tangible, même si elle ne s'inscrit pas forcément dans un registre purement chrétien.

Toujours est-il que Le Tramway est sans doute le texte le plus funeste de Claude Simon. La mort y est présente dans tous ses recoins. Chose que confirme Philippe Sollers : « et, en effet, il s'évade : de ses origines familiales, d'abord, et de leur couleur de mort. Voici l'agonie de sa mère dévote: "Tragique visage au bec de rapace, aspect à la fois guignolesque et macabre de marionnette". La Mort, grand personnage de tous ses livres, jusqu'au dernier, "le Tramway" » (Sollers, s.d.). Claude Simon, vieux et malade, semble être obsédé par l'idée de la Fin. Dans son texte, il met l'accent sur la mort. Il y développe une rhétorique qui se donne pour objectif de mettre en exergue ce thème. Pour le besoin de la démonstration, nous proposons détudier les éléments suivants : la ville, le mémorial de la mort, la maison familiale et les portraits.

\section{La ville}

La ville de Perpignan, qui est décrite dans ce texte et dont le nom est tu, est décrite comme un espace morbide. Il semble que le narrateur exhumant ses souvenirs d'enfance a, par une sorte de transfert, contaminé l'espace décrit par ses idées sinistres à la fin de sa vie. L'espace natalo-familial, qui est généralement décrit d'une manière euphorique, comme l'a constaté à ce propos Gaston Bachelard (voir 1960, p. 45), est dans Le Tramway un espace lugubre:

Quant à la ville, cétait comme si elle s’était répudiée elle-même (répudiant son église royale, le palais où avait couché Charles Quint, sa citadelle, les remparts dont plus tard Vauban l'avait entourée) pour ainsi dire exploser, seépanouir, accéder au sortir de ses étroites rues médiévales comme à une sorte d'antithèse d'elle-même sous les aspects d'une modernité d'ailleurs presque aussitôt fanée, déjà désuète et fragile [...]. (2001, p. 79)

Ce qui ajoute à l'aspect funeste de cette ville est l'aura de mort qui l'entoure et qui est associée à l'air. Il semble que toute la ville soit entourée d'un voile de mort qui menace de happer tous les habitants : 
Comme si quelque chose de plus que l'été n'en finissait pas d'agoniser dans l'étouffante immobilité de l'air où semblait toujours flotter ce voile en suspension qu'aucun souffle d'air ne chassait, s'affalant lentement, recouvrant d'un uniforme linceul les lauriers touffus, les gazons brûlés par le soleil, les iris fanés et le bassin d'eau croupie sous une impalpable couche de cendres, l'impalpable et protecteur brouillard de la mémoire. (2001, p. 141)

Dans ce passage, le champ lexical dominant est incontestablement celui de la mort. Les mots " agoniser ", " linceul » et " cendres " sont révélateurs dans ce sens. Sans compter les expressions qui réfèrent à l'idée de la mort mais symboliquement, notamment " étouffante immobilité », " voile en suspension ", " s'affaler », " recouvrant d'un uniforme » et "impalpable couche ». Or, il est à constater que cette description fait étrangement écho à ce passage de La Curée d'Emile Zola :

Un lent crépuscule tombait comme une cendre fine [...]. Et il tombait de ces hauteurs pâlissantes une telle mélancolie d'automne, une nuit si douce et si navrée, que le Bois, peu à peu enveloppé dans un linceul d'ombre, perdait ses grâces mondaines, agrandi [...]. Tout allait en se mourant. Dans l'effacement universel, au milieu du lac, la voile latine de la grande barque de promenade se détachait, nette et vigoureuse, sur la lueur de braise du couchant. (Zola, 2012, p. 15)

Dans cet extrait, la référence à la mort est explicite. Situé à l'orée du roman, il joue le rôle d'une mise en abyme prospective qui annonce la mort du personnage principal Renée. Il semble que, par le recours à l'intertextualité, Claude Simon, lui aussi, prédit la mort du personnage central de son texte, c'est-à-dire lui-même.

Il est à noter que dans la ville de Perpignan la mort circule dans les rues. Elle est associée à ses moindres éléments et se retrouve dans tous ses recoins, comme en témoigne le passage suivant :

[...] puis s'éloignaient peu à peu avant de mourir entre les pins, les poussiéreux buissons de lauriers et les massifs bordés d'iris dans le jardin où couchée comme une sorte d'épouvantail sur cette liseuse recouverte de cretonne à fleurs, que selon les heures on déplaçait sur le gravier en suivant la marche des ombres, maman agonisait lentement. (Simon, 2001, p. 89)

Dans ce passage, la végétation agonise elle aussi comme si elle était contaminée par l'agonie lente et tragique de la mère. En voici un autre exemple :

[...] et, les jours de vent, comme si quelqu'un essayait de le forcer, le rêche raclement contre le grillage de la fenêtre de l'extrémité d'une branche du dernier de la rangée de ces arbres un peu sinistres, moitié cyprès, moitié cèdres, qui, le long du mur d'enceinte, abritaient le jardin. (Simon, 2001, p. 107) 
Ces arbres " un peu sinistres " deviennent un symbole de la mort. D’ailleurs, il est fort éloquent que Le Tramway se termine par la description des fruits mûrs ratatinés :

[...] personne non plus, sauf les enfants, ne faisait attention aux figues trop mûres, à la peau ratatinée et ridée, presque noire, à la chair éclatée, pourpre, granuleuse et sucrée, éparpillées quelques mètres plus loin parmi les touffes d'herbe. (2001, p. 140)

L’été, qui fait peser sur la ville un air étouffant qui ressemble à un voile morbide, suggère sans ambages l'idée de la mort. L'auteur semble vouloir dire que les enfants sont les seuls à être sensibles à l'omniprésence de la mort. C'est de cette manière que Claude Simon enfant savait secrètement que la mort de sa grand-mère ainsi que celle de sa mère étaient imminentes.

Soulignons également que même le cirque, qui normalement apporte à un enfant beaucoup de bonheur et de joie, dans la mesure où il se présente pour celui-ci comme un espace de découverte, de curiosités et surtout de distraction sans limite, est, dans Le Tramway, un espace morbide :

[...] lieu (l'ensemble à l'apparence délabrée de ces baraquements passés au goudron) qui, par une sorte d'ironique paradoxe, portait le nom de « Plage Mondaine » et qui (remplacé plus tard, mais moins près de la mer et presque à côté du terminus du tramway, par un sommaire cube de béton peint en jaune et baptisé « Casino »), quoique assez lugubre d'aspect [...]. (2001, p. 2)

Dans cet espace lugubre, le temps ne peut être que maussade :

[...] entre les baraques de la foire installée chaque automne sous les platanes qui longeaient l'Allée des Marronniers et où, dans le mélancolique crépuscule clignotaient les lumières tentatrices des manèges et des attractions dont la principale [...]. (2001, p. 34)

À l'encontre du crépuscule qui, chez les poètes romantiques, un moment d'effusion des sentiments et de jouissance de la présence de la bien-aimée, est dans Le Tramway taciturne irrémédiablement. Les malades dans les ambulances, qui les conduisent à l'hôpital, vivent douloureusement cette tranche du temps :

[...] ils pouvaient voir s'enfuir vertigineusement dans la grisaille du crépuscule la succession confuse des façades, des carrefours, de feux rouges, de devantures et de cafés illuminés, tout comme aspiré, surgissant de cet inconnu vers lequel ils étaient emportés, basculant, s'enfuyant et disparaissant dans une sorte d'entonnoir, d'insondable et noire perspective. (2001, p. 30) 
En somme, la ville est présentée comme un espace lugubre. Tout ce qui s'y rapporte est associé à la mort, à tel point qu'il n'est pas exagéré de dire que la mort dans Le Tramway de Claude Simon est le personnage le plus important.

\section{Le mémorial de la mort}

Le monument érigé à Perpignan en l'honneur des victimes de la Première Guerre mondiale occupe une place de choix dans les premiers chapitres du Tramway. Pour bien jouer son rôle, ce mémorial est placé à l'entrée du jardin public :

Monument en grès rose du pays et aussi haut qu'une maison de deux étages, élevé en bordure du jardin public et où sur un fond de marbre noir (comme une page de deuil encadrée d'un matériau précieux) s'allongeaient les interminables colonnes de noms dorés à la feuille des morts de la ville [...]. (2001, p. 35)

De par l'emplacement qui lui a été choisi, le mémorial devient, en quelque sorte, le moyeu autour duquel s'organise la ville. Tous les déplacements passent près de lui et par lui, notamment ceux effectués par le biais du tramway :

[...] la motrice pénétrait dans la ville, descendait d’abord la longue pente qui menait au jardin public $[\ldots]$ tournait à gauche à hauteur du monument aux morts $[\ldots]$ pour s'immobiliser en fin de course, presque au centre-ville [...]. (2001, p. 14)

Ce mémorial, qui ressemble à plus d'un égard aux monuments érigés au Moyenâge par les catholiques pour rappeler aux fidèles que la mort est inéluctable, est également un lieu de rencontre des soldats que la guerre a estropiés ou privés d'une partie de leur corps :

[...] à partir du monument aux morts élevé à l'entrée du square municipal, semblait être, l'après-midi (comme s'il y avait un lien entre le monumental monument et eux), le rendez-vous d'une demi-douzaine de ces voiturettes constituées d'un siège d'osier peint en noir, $[\ldots]$ et actionnée par les mains de ces personnages [...] que maman appelait avec aurait-on dit une sorte de joie mauvaise d'un nom composé (les hommes-troncs) [...]. (2001, p. 19-20)

Nous pouvons inférer que le mémorial de la mort, situé au centre de la ville, renforce l'aura de mort, qui semble envelopper la ville d'un voile de mort. 


\section{La maison familiale}

Cette demeure est décrite comme un espace funeste. " Dans cette maison, [Claude Simon] assistera $[\ldots]$ au décès de sa grand-mère maternelle. L'image que va garder notre auteur de son espace natal à Perpignan [...] est bien évidemment une image macabre et de morbidité » (Belarbi, 2016, p. 403). La maison familiale a les attributs du cimetière : elle est vaste, sombre et un silence de mort y règne :

[...] traditionnelles lamentations de ma tante que ce retour annuel plongeait dans un ostentatoire abattement chaque fois renouvelé lorsque après les quatre mois passés à la campagne elle se retrouvait dans ce qu'elle appelait son "tombeau ", c'est-à-dire le vaste appartement qui, quoique donnant sur de spacieuses cours et un spacieux jardin, était, il est vrai, assombri par les rameaux d'un gigantesque acacia [...]. (Simon, 2001, p. 89-90)

D'ailleurs, par une étrange coïncidence, le retour à la demeure familiale après le séjour habituel de quatre mois passé dans la maison de la campagne s'effectue le 2 novembre. En d'autres termes, il se réalise le jour de tous les fidèles défunts :

[...] retour qui, coïncidant avec le jour des Morts, était chaque année le prétexte pour ma tante (qui pourtant y était née et y avait toujours vécu) d'afficher ce hautain désespoir. (Simon, 2001, p. 89-90)

Donc, à l'encontre de la maison familiale des romantiques qui est un espace hautement euphorique, la maison où Claude Simon a passé une partie de son enfance, est un lieu morbide.

\section{Les portraits}

D’une manière générale, les portraits brossés par Claude Simon sont lacunaires. Ils ne sont pas suffisamment détaillés et ne touchent pas l'ensemble des éléments du corps et, plus particulièrement, du visage, pour donner une description quasi-complète du personnage. Si l'auteur se refuse obstinément à produire des portraits physiques à la Balzac, par exemple, il met l'accent, en revanche, sur les éléments qui, pour lui, sont suffisants pour décrire le personnage. Lorsqu'il s'agit de décrire les moribonds, l'un des éléments du visage qu'il met généralement en valeur est le nez. Il semble que pour ce dernier, le nez, lorsqu'il devient proéminent, est l'indice de la mort prochaine. C’est pour cela que cet élément est ce que sa mémoire retient, entre autres, de sa mère agonisante : 
[...] jusqu'au jour où je l'ai vue pour la dernière fois - encadrant un semblable et tragique visage au bec de rapace, comme si jeetais condamné à ne voir de la mort, du moins celle que l'on appelle " naturelle " (ou de son approche), que cet aspect à la fois guignolesque et macabre de marionnette [...]. (Simon, 2001, p. 63)

Il en va de même avec un vieillard qui était hospitalisé en même temps que lui et des rescapés de la Première Guerre mondiale que sa mère avait tendance à appeler les hommes-troncs :

L'autre occupant de la chambre double où l'on roula mon lit après la nuit passée au TRANSIT était un vieillard dont la première chose qui me frappa (comme si le souvenir des hommes-troncs et de ma mère associait à la souffrance et à la mort l'image de ces nez osseux en bec d'aigle dont l'amaigrissement causé par la maladie et par l'âge finissait par en faire, au détriment de tous les autres, le principal élément d'un visage) fut un de ces nez proéminents et pour ainsi dire acérés qui semblait avoir effacé tout autre trait [...]. (2001, p. 47)

Dans cette citation, l'association du nez à la mort est éloquente. Si la madeleine trempée dans un verre de thé est pour le narrateur du Du côté de chez Swann associée à Combray, pour Claude Simon, le nez osseux au milieu d'un visage amaigri par les lacérations du temps et par celles de la maladie est intimement lié à la mort.

Lorsque le personnage simonien n'est pas une allégorie de la mort, il se trouve associé à la mélancolie. C'est le cas de l’oncle Charles :

[...] un masque d'aimable cynisme derrière lequel se cachait quelque secrète mélancolie que ne pouvaient consoler ni le ridicule château ni la grosse automobile rangée au côté des autres [...]. (2001, p. 101)

C'est aussi le cas du conducteur et du receveur du tramway qui ont « les visages taciturnes et maussades » $(2001$, p. 19).

En somme, la plupart des personnages simoniens sont associés à la mort. Ils ont des aspects lugubres, taciturnes et morbides. Ils sont décrits comme des morts en puissance.

$\mathrm{Au}$ terme de ce chapitre, nous pouvons arguer que la mort se trouve dans tous les recoins du Tramway de Claude Simon. Elle le traverse de bout en bout. Nous sommes même en droit de dire qu'elle est le personnage principal de cette " autothanatographie ». Un nombre considérable d'éléments évoquent explicitement cette omniprésence de la mort, en l'occurrence la ville, le mémorial de la mort, la maison familiale et les portraits des personnages. 


\section{Ars moriendi}

Comme nous l'avons déjà souligné, Claude Simon a toujours refusé qu'on attribue à son œuvre une quelconque portée didactique. Dans le discours qu'il a tenu lors de la cérémonie de remise du prix Nobel à Stockholm, il a réitéré ce refus en affirmant : " et cependant, je n'ai jamais encore, à 72 ans, découvert aucun sens à tout cela, si ce n'est, comme l'a dit, je crois, Barthes, après Shakespeare, que "si le monde signifie quelque chose, c'est qu'il ne signifie rien - sauf qu'il est” " (Simon, 1986, p. 24).

Or, à considérer le texte objet de notre corpus, nous constatons au moins deux choses : de prime abord que les prédicats constatifs sont d'entre tous les moins dominants dans le texte. Ce qui est bizarre pour un auteur qui de son propre dire se contente simplement de constater l'existence du monde. Ensuite, la référence au retour au primordial a pratiquement disparu du Tramway. En effet, dans les textes antérieurs, notamment dans Les Géorgiques, L'Acacia et Le Jardin des plantes, le travail ravageur et destructeur de la guerre est associé au retour à l'ordre des choses que Claude Simon appelle le primordial. Ce n'est pas par hasard si le numéro de la revue Critique, consacré à l'auteur en 1981, porte le titre : « La Terre et la guerre dans l'œuvre de Claude Simon ". Dans l'imaginaire simonien, ces deux facteurs sont intimement liés. Loin de se contredire, ils se rejoignent. Comme le remarque, à juste titre, l'oncle Charles : " quoique si l'on y réfléchit les deux choses ne soient pas tellement contradictoires. Je veux dire en ce qui concerne les qualités requises. Je veux parler de cet éternel recommencement» (Simon, 1981, p. 449).

Dans Le Tramway, Claude Simon expose une nouvelle conception du monde, et, plus précisément, de son rapport au monde. La mort n'est plus un ordre naturel du monde. Elle n'est plus la fin d'un lent processus qui est la vie. Elle n'est plus ce qui permet la régénération des choses. Elle n'est plus ce qui préside au renouvellement du monde. La mort, dans Le Tramway, devient tragique. Elle est létau qui enserre les vivants, les étouffe et les accable sans rémission et sans répit. Claude Simon, de plus en plus démuni et fragile par la maladie et surtout par la vieillesse, vit l'angoisse de la mort comme l'a vécue avant lui Eugène Ionesco qui, à la fin de sa vie, a invité les chercheurs à « réfléchir sur la mort » (Ionesco, 1991, p. 98).

Derrière sa nouvelle conception sur la mort se laisse entrevoir un fort sentiment de l'inanité de la vie. Encerclé par la mort, qui, dorénavant hante son esprit et ses pensées, l'auteur se rend compte que le monde est vain, futile et inutile. Dans Le Tramway, il expose son art de la mort qui est intimement liéà la vanité. C'est à ce niveau qu'il peut enfin se soustraire à l'emprise de la pensée structuraliste et de la pensée moderne qui l'ont influencé pendant une longue période. Sentant ses forces choir et l'abandonner de plus en plus, l'auteur se convertit à l'humanisme. Non pas un humanisme moralisateur ou idéalisé, c'est-à-dire qui se présente sous les attraits romantiques ou qui sublime la vie, mais tout simplement devient homme, un homme qui sent, qui a des affects et une sensibilité. 
La vanité du monde est donc présentée dans Le Tramway sous un autre jour, par d'autres techniques. Dans ce volet, nous proposons d'étudier quelques procédés auxquels recourt Claude Simon pour présenter sa nouvelle perception du monde. En effet, à l'instar des poètes baroques, lécrivain recourt à un ensemble de symboles pour exposer la vanité de la vie, en l'occurrence : le tramway, la cigarette, l'hôpital et la maladie.

\section{Le tramway}

Dans le texte de Claude Simon, le tramway n'est pas un simple moyen de locomotion qui a marqué son enfance. Il est plus important que cela comme l'indique, entre autres, le fait que l'auteur ait choisi de titrer son texte Le Tramway. Il se trouve imbriqué dans un réseau d'images qui symbolisent entre autres la mort. Dans ce vaste réseau, au tramway incombe la fonction de signifier l'idée d'un prompt passage entre la vie et la mort. Il devient, dans le texte simonien, en quelque sorte, une représentation moderne de la barque de Charon conduisant les morts au Royaume d'Hadès. D’ailleurs, la référence à ce mythe est explicite :

[...] et que le sculpteur (apparenté à la riche famille qui produisait un apéritif réputé) avait disposés non pas sur un rang mais selon un léger arc de cercle comme si, insoucieux des passants ou des spectateurs, ils étaient là non pour se montrer mais comme sortis du royaume des morts et converser entre eux [...]. (Simon, 2001, p. 35)

L'image du tramway, comme embarcation séparant les deux mondes, celui des vivants et celui des morts, est renforcée, notamment, dans le passage suivant :

Si le terminus pour ainsi dire domiciliaire de la ligne du tramway se situait presque au cœur de la ville, par contre, à son autre extrémité, les rails couverts de rouille disparaissaient, quelques mètres après un butoir, sous une couche de sable dont le vent de mer les recouvrait avec la même patiente obstination [...]. (Simon, 2001, p. 40)

Cette image est fort éloquente. La référence au royaume des morts y est plus évidente. Il semble que le tramway termine son chemin en se glissant à l'intérieur de la terre. Soulignons également la référence à la mer. Il faut traverser le Styx pour enfin parvenir au Royaume d'Hadès.

Il n'est pas étonnant que le terminus du tramway se trouve à proximité de ce que l'auteur appelle " le monument aux morts ». Il n'est pas surprenant également que le terminus soit situé au centre de la ville. 


\section{La cigarette}

Cette image retient l'attention, parce qu'elle occupe une plage descriptive assez importante. En voici un fragment :

[...] le conducteur (le wattman) la tête fortement inclinée pour éviter la flamme du briquet rasant ses lèvres, tirait une dernière bouffée de ce mégot réduit à moins d'un centimètre de papier dentelé de noir qui rougeoyait un instant avant d'être délicatement saisi entre deux doigts, arraché de la lèvre où il avait fini par se coller, et enfin jeté. (Simon, 2001, p. 18)

De la première phase qui commence par allumer une cigarette jusqu'à la dernière phase où on jette le mégot s'écoule peu de temps. La cigarette s'associe parfaitement à l'idée de l'éphémérité. Soulignons également que la cigarette évoque le souffle et le vent qui sont, comme l'a magistralement montré le grand spécialiste du baroque Jean Rousset, des motifs fort usités dans l'œuvre poétique baroque ${ }^{3}$. La cigarette devient, dans ce texte de Claude Simon, la métaphore de l'évanescence et de la fugacité. La vie s’écoule avec une rapidité ahurissante et il en va de même avec la cigarette.

\section{L'hôpital}

Il faut noter qu'à l'hôpital l'auteur réserve toute une séquence, qui est obsessionnelle dans Le Tramway. Loin d'être un simple espace lié à des souvenirs douloureux, la structure hospitalière accède au statut de symbole. D’ailleurs, l'auteur en est conscient, puisqu'il note à ce propos :

Entouré de tous côtés par l'anarchique tissu urbain au sourd grondement, l'hôpital [...] comme une sorte d'entité en soi, d'univers en réduction, fermé sur lui-même, ripoliné et fini, du service d'obstétrique à la morgue, offrant comme en raccourci (ou en condensé) les successifs états de la machine humaine de la naissance à l'agonie en passant par toutes les déviations et anomalies possibles jusqu’à sa définitive corruption. (Simon, 2001, p. 105)

Cette métaphore de la vie humaine courte, endolorie et se terminant par la mort qu'est l'hôpital est originale. Aucun autre écrivain, du moins à notre connaissance, n'a établi un lien entre la fugacité de la vie en tant que processus, qui passe, certes, par un certain nombre d'état, mais demeure quand même d'une extrême brièveté.

3. Voir, entre autres, J. Rousset. (1988). Anthologie de la poésie baroque française [1961]. Paris, France : Corti. 


\section{La maladie}

Comme pour l'hôpital, la maladie, qui est fort présente dans la poésie baroque, devient sous la plume de Claude Simon l'image de l'évanescence et de la concision. Elle est associée au processus infernal que représente la vie. Le personnage malade passe par une série d'états avant d'arriver au stade final, qui est la mort. Cette image est filée à travers l'histoire de la mère. Dans Le Tramway, cette dernière est décrite comme une allégorie de la mort certes, mais elle est l'image de la fugacité :

Son propre visage, donc, depuis que la maladie qui devait l'emporter s'était attaquée à elle, sétait mis, comme par une sorte de mimétisme (ou de coquetterie macabre) à tout d'abord simplement maigrir, pour ensuite se creuser, se momifier peu à peu. (Simon, 2001, p. 22)

Il est vrai que la maladie, qui devait emporter la mère, lui a laissé quelques années à vivre, mais, dans l'imaginaire de l'auteur, ces quelques années ne représentent qu'un court instant. D'ailleurs dans l'exemple que nous venons de citer, le rythme saccadé des propositions et le recours à une seule phrase, pour résumer le lent processus de la maladie crée chez le lecteur le sentiment que la maladie de la mère fut foudroyante.

La description de la dégénérescence, qui s'abat sur la mère, suite à sa maladie, et qui ressemble à plus d'un égard à l'autoportrait que brosse Pierre de Ronsard dans son poème intitulé Je n'ai plus que les os, devient donc l'image du passage et de l'éphémère. Elle est tout à fait le contraire de ce que représente la grand-mère. Pour l'auteur, «la vieille veuve éplorée au camée pompéien » comme il l'appelle dans Les Géorgiques, représente la rigidité et la fixité, mais de la dernière phase de la vie :

[...] grand-mère dont, effaçant toute autre image, il me semble toujours voir le masque de tragédie, blafard et saupoudré de gris, flottant, impondérable et, avec ses poches soulignant les yeux, ses chairs affaissées et sa bouche aux coins tombants, comme suspendu dans le vide, au long du dédale des couloirs obscurs, empreint d'une affliction mêlée d'hébétude, comme l'image même du malheur ou plutôt d'une somme de malheurs où s'additionnaient sans doute pêle-mêle la perte de son mari (notre grand-père) suivie de celle de sa fille aînée [...]. (Simon, 2001, p. 90)

N'ayant pas été malade, le corps de la grand-mère n'est pas passé par des états successifs de dégénérescence. Quand l'auteur l'a connue, elle était déjà vieille et donc, pour lui, elle n'est pas symbole du changement et de la transformation. Tout au contraire, elle a toujours symbolisé, dans son imaginaire, la persistance et la durée. Considérons encore le passage suivant : « ... simplement quand je suis revenu pour les grandes vacances après Pâques la liseuse n’était plus là je me rappelle que sans rien dire je l'ai cherchée $[\ldots]$ » (Simon, 2001, p. 68). Cet extrait met certes en valeur la soudaineté 
de la mort de la mère, mais permet à l'auteur de filer sa métaphore en suggérant également, par la disparition de la liseuse, la vanité de la vie. Le temps, qui emporte les êtres humains, efface leurs traces. Il est étrange que Claude Simon n'ait pas réagi à cette idée en proposant l'image de la durabilité et de la persistance que permet, par exemple, l'art. C'est vraiment bizarre d'autant plus qu'il a été profondément influencé par Marcel Proust et qui, comme ce dernier, s'est lancé dans l'aventure de la recherche du temps perdu. Il semble que dans Le Tramway, l'auteur ait pris à ce niveau ses distances par rapport à son modèle. Il a fini par comprendre que l'art ne récupère rien. Et c'est dans cette brèche que s'insinue le tragique de la pensée de Claude Simon.

$\mathrm{Au}$ terme de cette étude, nous pouvons constater que le thème de la vanité est au centre du Tramway de Claude Simon. Ce thème est certes primordial dans l'imaginaire de cet auteur et nous le retrouvons dans l'ensemble de son œuvre, mais, à notre avis, un changement au niveau de la perception de cette vanité et de sa présentation s'est opéré avec Le Tramway.

Dans ce texte, qui est le dernier de son œuvre et qui a été écrit quatre années avant son décès, Claude Simon, sans doute obsédé par la mort, a présenté une vision postmoderne de la vanité. En effet, nous y constatons un rejet de la vision matérialiste de la mort qui a trouvé son summum dans Les Géorgiques et, selon laquelle, l'auteur pense que la guerre, tout comme l'agriculture, est un phénomène purement cosmique et que, de ce fait, l'ordre qui prévaut est celui du retour des choses à leur origine : la terre.

Dans Le Tramway, au contraire, l'auteur évoque la dimension tragique du temps et surtout de son passage accéléré et irrémédiable. Ce dernier a également renié sa conception transcendantale de l'art d'une manière générale et de la littérature en particulier. Rien ne résorbe le travail ravageur du temps semble être le message qu'il a cherché à faire passer en filigrane. Il a voulu attirer l'attention sur la condition de l'individu en tant que mortel. Il a repris à son compte le memento mori des poètes catholiques de leépoque médiévale et a présenté une nouvelle perception de la finitude à travers le recours à une série d'images fortement symboliques à ce niveau.

\section{RÉFÉRENCES}

Bachelard, G. (1960). Poétique de la rêverie. Paris, France : PUF.

Belarbi, M. (2016). Lieux de mémoire et écriture dans l’ouvre de Claude Simon. Histoire de l'écriture et écriture de l'H(h)istoire. Toulouse, France : Les Presses Universitaires.

Calle-Gruber, M. (2011). Claude Simon : une vie à écrire. Paris, France : Seuil.

Ionesco, E. (1991). Notes et contre-notes. Paris, France : Folio.

Rousset, J. (1988). Anthologie de la poésie baroque française [1961]. Paris, France : Corti.

Simon, C. (1981). Les Géorgiques. Paris, France : Minuit.

Simon, C. (1986). Discours de Stockholm. Paris, France : Minuit.

Simon, C. (avril 1998). Entretien. Scherzo, 3. 5-11. 
Simon, C. (1999). Entretien. Genesis, 13. 115-121.

Simon, C. (2001). Le Tramway. Paris, France : Minuit.

Sollers, Ph. (s.d.). Consulté sur http://www.philippesollers.net/claude-simon.html

Zola, E. (2012). La Curée. Paris, France : Livre de poche.

RÉSUMÉ : Le thème de la vanité occupe une place de choix dans Le Tramway de Claude Simon. Dans ce texte, il a adopté une nouvelle conception de la vanité. Certes, l'auteur a toujours évoqué des thèmes intimement associés à la vanité, tels que le thème de la mort, le thème de la mélancolie, le thème de la guerre, etc., mais la finitude a toujours été pour lui une phase cruciale pour le retour au primordial et à l'origine, pour le commencement d'un nouveau cycle de la vie. Dans Le Tramway, la vanité du monde est présentée d'une manière tragique. Il n'y a aucune référence à la primordialité ni au retour cyclique des choses. L'auteur insiste sur l'actualisation, dans la mémoire, du temps de la mort. Un nombre important de métaphores et de symboles mettent en exergue ce thème de la mort ; c'est comme si l'auteur lançait au lecteur un memento mori de la part de quelqu'un sur le point de vivre cette expérience funeste qui est irrémédiable et définitive. C'est ce qui confère au texte une dimension tragique et humaine indéniable.

Mots-clés : vanité postmoderne tragique, mélancolie, memento mori, ars moriendi

\section{Claude Simon's Tramway: a postmodern vanity?}

ABSTRACT: The theme of vanity occupies a prime place in Claude Simon's Tramway. In this text, he has adopted a new conception of vanity. Certainly, the author has always evoked themes intimately associated with vanity, such as the theme of death, the theme of melancholy, the theme of war, etc., but the "finitude" has always been for him a crucial phase for the return to the primordial and the origin for the beginning of a new cycle of life. In Tramway, the vanity of the world is presented in a tragic way. No reference to the primordiality or the cyclical return of things. The author insists on updating in the memory of the time of death. A large number of metaphors and symbols highlight this theme of death; it is as if the author throws to the reader a memento mori from someone on the point of experiencing this fatal experience which is irremediable and definitive. This gives the text a tragic and undeniable human dimension.

Keywords: vanity postmodern tragic, melancholy, memento mori, ars moriendi 